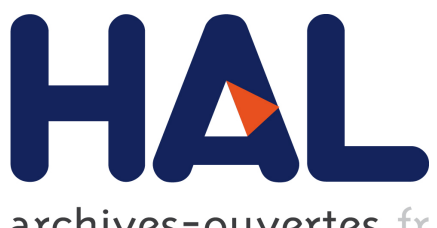

archives-ouvertes

\title{
Primal Infon Logic with Conjunctions as Sets
}

\author{
Carlos Cotrini, Yuri Gurevich, Ori Lahav, Artem Melentyev
}

\section{To cite this version:}

Carlos Cotrini, Yuri Gurevich, Ori Lahav, Artem Melentyev. Primal Infon Logic with Conjunctions as Sets. 8th IFIP International Conference on Theoretical Computer Science (TCS), Sep 2014, Rome, Italy. pp.236-249, 10.1007/978-3-662-44602-7_19 . hal-01402046

\section{HAL Id: hal-01402046 \\ https://hal.inria.fr/hal-01402046}

Submitted on 24 Nov 2016

HAL is a multi-disciplinary open access archive for the deposit and dissemination of scientific research documents, whether they are published or not. The documents may come from teaching and research institutions in France or abroad, or from public or private research centers.
L'archive ouverte pluridisciplinaire HAL, est destinée au dépôt et à la diffusion de documents scientifiques de niveau recherche, publiés ou non, émanant des établissements d'enseignement et de recherche français ou étrangers, des laboratoires publics ou privés. 


\title{
Primal Infon Logic with Conjunctions as Sets
}

\author{
Carlos Cotrini $^{1}$, Yuri Gurevich ${ }^{2}$, Ori Lahav ${ }^{3}$, and Artem Melentyev ${ }^{4}$ \\ 1 Swiss Federal Institute of Technology \\ 2 Microsoft Research \\ 3 Tel Aviv University \\ 4 Ural Federal University
}

\begin{abstract}
Primal infon logic was proposed by Gurevich and Neeman as an efficient yet expressive logic for policy and trust management. It is a propositional multimodal subintuitionistic logic decidable in linear time. However in that logic the principle of the replacement of equivalents fails. For example, $(x \wedge y) \rightarrow z$ does not entail $(y \wedge x) \rightarrow z$, and similarly $w \rightarrow((x \wedge y) \wedge z)$ does not entail $w \rightarrow(x \wedge(y \wedge z))$. Imposing the full principle of the replacement of equivalents leads to an NP-hard logic according to a recent result of Beklemishev and Prokhorov. In this paper we suggest a way to regain the part of this principle restricted to conjunction: We introduce a version of propositional primal logic that treats conjunctions as sets, and show that the derivation problem for this logic can be decided in linear expected time and quadratic worst-case time.
\end{abstract}

\section{Introduction}

Propositional infon logic is a version of propositional multimodal intuitionistic logic [7. It is applicable for policy and trust management but the derivability problem for propositional infon logic is PSPACE-complete. Nevertheless, an expressive fragment of this logic, called propositional primal infon logic (PIL, in short), is decidable in linear time [7]. PIL is far below propositional infon logic in the time-complexity hierarchy. A natural problem arises how to extend the expressive power (and usefulness) of PIL keeping the logic feasible. In this paper, we present substantial progress toward this goal.

One of the main limitations of PIL is that it does not satisfy the principle of replacement of equivalents, that allows us to substitute a formula with an equivalent one in any context. For example, the formulas $x \wedge y$ and $y \wedge x$ are equivalent in PIL (i.e., each one is derivable from the other). However, $(x \wedge y) \rightarrow z$ and $(y \wedge x) \rightarrow z$ are not. In general, replacing a variable occurring in some formula with $x \wedge y$ is not the same as replacing it with $y \wedge x$. A similar situation occurs, e.g., with formulas of the form $(x \wedge y) \wedge w$ and $x \wedge(y \wedge w)$. 
Imposing the full principle of replacement of equivalents on PIL makes it NP-hard [2]. Nevertheless, in this paper, we present an extension of PIL, called SPIL, that overcomes this limitation for conjunction. The idea behind SPIL is to treat conjunctions as sets of conjuncts (the ' $\mathrm{S}$ ' in SPIL alludes to the word "set"). In other words any two conjunctions are viewed equivalent if the sets (not multisets!) of their conjuncts are the same, and the reasoning is done modulo this equivalence. For example, this equivalence relation identifies formulas $(x \wedge y) \rightarrow z$ and $(y \wedge x) \rightarrow z$.

This paper is organized as follows. First, we recall the syntax of PIL (Section 2). Then we define SPIL (Section 3), and we prove the local formula property for its Hilbertian calculus: any derivation of a formula $X$ from a set $\Omega$ of formulas can be modified so that it uses only a small set of "local formulas" computable from $\Omega \cup\{X\}$. In Section 3.1 we present a Kripke-style semantics for SPIL. Finally, in Section 4 , we present an efficient algorithm for the multi-derivability problem for SPIL. An implementation of the algorithm is available at http://dkal.codeplex. com/ (in the context of Distributed Knowledge Authorization Language [3]).

Related work. We refer the reader to detailed related work sections: subsection 1.1 in the article [1] on propositional primal logic with disjunction, and section 6 in the article [5] on extensions of PIL with transitivity of implication. In addition, we note that proof systems in which derivations are performed modulo an equational theory between propositions were studied earlier in different contexts (see, e.g., [6]).

\section{Preliminaries}

We start with describing PIL (propositional primal infon logic), originally presented in [7. We presume a set of propositional variables $\left\{v_{1}, v_{2}, \ldots\right\}$, a set of principal constants $\left\{p_{1}, p_{2}, \ldots\right\}$ and a constant $T$ (used to denote an item of information that is known to all principals). The formulas of PIL are built from the propositional variables and $T$ using the binary connectives $\wedge, \vee, \rightarrow$, and unary connectives of the form "q:" (called: quotations) where $q$ ranges over principal constants. The intended meaning of a formula $q: x$ is that: the principal $q$ said $x$. The size $s z(x)$ of a formula $x$ is taken to be the number of connectives occurring in $x$. For any sequence of principal constants $q_{1}, q_{2}, \ldots, q_{k}$, we call the string $\boldsymbol{q}=q_{1}: q_{2}: \ldots q_{k}$ : a quotation prefix (where $\epsilon$, the empty sequence, is a quotation prefix as well). 


\begin{tabular}{|c|c|c|c|c|c|c|c|}
\hline & $\top) \overline{\boldsymbol{q}^{\top}}$ & \multicolumn{2}{|c|}{$\boldsymbol{q}(x \wedge y)$} & $(\wedge \mathrm{e})$ & $\frac{\boldsymbol{q}(x \wedge y)}{\boldsymbol{q} x}$ & & $\frac{\boldsymbol{q}(x \wedge y)}{\boldsymbol{q} y}$ \\
\hline \multirow{2}{*}{$(\vee \mathrm{i})$} & $\boldsymbol{q} x$ & $\boldsymbol{q} y$ & \multirow[t]{2}{*}{$(\rightarrow \mathrm{i})$} & $\boldsymbol{q} y$ & \multirow{2}{*}{$(\rightarrow \mathrm{e})$} & $\boldsymbol{q} x$ & $\boldsymbol{q}(x \rightarrow y)$ \\
\hline & $\boldsymbol{q}(x \vee y)$ & $\boldsymbol{q}(x \vee y)$ & & $\boldsymbol{q}(x \rightarrow y)$ & & & $\boldsymbol{q} y$ \\
\hline
\end{tabular}

Fig. 1. Calculus for PIL. $\boldsymbol{q}$ ranges over quotation prefixes and $x, y$ over formulas.

Figure 1 provides a Hilbertian calculus defining PIL. For a set of formulas $\Gamma$ (called hypotheses), a derivation $D$ of a formula $x$ from $\Gamma$ in PIL is a finite tree such that each node $u$ is labeled with a formula $D(u)$. The root is labeled with $x$ and leaves are labeled with (instances of) axioms or formulas in $\Gamma$. If a node $u$ has children $u_{1}, u_{2}, \ldots, u_{n}$, then $D\left(u_{1}\right), \ldots, D\left(u_{n}\right) / D(u)$ is an instance of an inference rule. The size of the derivation is the number of nodes in this tree.

Given two sets of formulas $\Gamma$ and $\Delta$, the problem of deciding which formulas in $\Delta$ are derivable from $\Gamma$ in PIL is called the multi-derivability problem for PIL. This problem is decidable in linear time [4,7].

\section{The Logic SPIL}

We present an extension of PIL that we call SPIL. The letter 'S' alludes to the word "set" and reflects our intention to treat conjunctions as sets of conjuncts. To define SPIL we use an auxiliary notion of abstract formulas.

Definition 1. An equivalence relation $\sim$ between formulas is defined as follows: $x \sim y$ if $x$ and $y$ are related according to the reflexive transitive symmetric closure of the rewriting relation induced by the following term rewriting system: $:^{5}$

$-\left(x_{1} \wedge x_{2}\right) \longrightarrow\left(x_{2} \wedge x_{1}\right)$

$-\left(\left(x_{1} \wedge x_{2}\right) \wedge x_{3}\right) \longrightarrow\left(x_{1} \wedge\left(x_{2} \wedge x_{3}\right)\right)$

$-\left(x_{1} \wedge x_{1}\right) \longrightarrow x_{1}$

$$
\begin{aligned}
& -\left(x_{1} \wedge \top\right) \longrightarrow x_{1} \\
& -q:\left(x_{1} \wedge x_{2}\right) \longrightarrow\left(q: x_{1}\right) \wedge\left(q: x_{2}\right) \\
& -q: \top \longrightarrow \top
\end{aligned}
$$

Roughly speaking, we have $x \sim y$ if $x$ and $y$ are the same formulas modulo the following properties of $\wedge$ : commutativity, associativity, idempotence, contraction of the identity element $T$, as well as the distributivity of quotations over $\wedge$.

Example 1. The formula $\left(v_{1} \rightarrow p_{1}:\left(\left(p_{1}: v_{1}\right) \wedge v_{2}\right)\right)$ is equivalent to $\left(\left(\left(v_{1} \wedge v_{1}\right) \wedge \top\right) \rightarrow\left(p_{1}: v_{2} \wedge\left(p_{1}: p_{1}: v_{1}\right)\right)\right)$.

\footnotetext{
${ }^{5}$ Recall that rewriting rules of a term rewriting system may be applied under any context, and not necessarily on the topmost level.
} 
Definition 2. Abstract formulas are equivalence classes of formulas under $\sim$. The size $s z(X)$ of an abstract formula $X$ is defined as $\min \{\mathrm{s} z(x) \mid x \in X\}$.

We use $X, Y, \ldots$ as metavariables for abstract formulas. The equivalence class of a formula $x$ under $\sim$ is denoted by $[x]$. Since abstract formulas play a dominant role in SPIL, we will refer to them simply as formulas, where true (non-abstract) formulas will be called concrete formulas. We define several operations on formulas.

Definition 3. For two formulas $X, Y$ and connective $* \in\{\rightarrow, \vee\}$, $X * Y:=[x * y]$ for some $x \in X$ and $y \in Y$. Similarly, for a formula $X$ and a quotation prefix $\boldsymbol{q}, \boldsymbol{q} X:=[\boldsymbol{q} x]$ for some $x \in X$.

\section{Definition 4.}

- A formula $X$ is called conjunctive if $X=[\top]$ or $X=[x \wedge y]$ for concrete formulas $x, y$ satisfying $x \not y, x \neq \top$ and $y \not \top$.

- A finite set of non-conjunctive formulas with at least two elements is called a conjunction set.

- For a conjunction set $S, \wedge S:=\left[\left(\cdots\left(\left(x_{1} \wedge x_{2}\right) \wedge x_{3}\right) \ldots\right) \wedge x_{n}\right]$ for some concrete formulas $x_{1}, \ldots, x_{n}$ such that $S=\left\{\left[x_{1}\right], \ldots,\left[x_{n}\right]\right\}$.

It is easy to see that these operations are well-defined. In particular, the choices of concrete formulas is immaterial. Note that we use conjunction sets rather than multisets, and that, by definition, conjunction sets contain at least two members.

Proposition 1. $\bigwedge S$ is conjunctive for every conjunction set $S$.

The next proposition allows us to use inductive definitions and prove claims by induction on size of formulas.

Proposition 2. Every formula $X$ is either non-conjunctive and exactly one of the following holds:

- $s z(X)=0$ and $X=[v]$ for a unique propositional variable $v$.

- $X=Y * Z$ for unique formulas $Y$ and $Z$ and $* \in\{\rightarrow, \vee\}$. In this case $s z(X)=s z(Y)+s z(Z)+1$.

$-X=q: Y$ for unique principal constant $q$ and formula $Y$. In this case $s z(X)=s z(Y)+1$, and $Y$ is non-conjunctive.

or else $X$ is conjunctive and either $s z(X)=0$ and $X=[\top]$, or $X=\bigwedge S$ for a unique conjunction set $S$. In the latter case, $s z(Y)<s z(X)$ for every $Y \in S$. 


\begin{tabular}{|cc|}
\hline$(\tilde{\mathcal{T}}) \frac{}{[\mathrm{T}]} \quad(\tilde{\wedge} \mathrm{i}) \frac{X_{1} \quad X_{2} \quad \ldots}{X_{n}}$ & $X_{n}$ \\
$(\tilde{\wedge} \mathrm{e}) \frac{\Lambda S}{X}$ where $S=\left\{X_{1}, \ldots, X_{n}\right\}$ and $n \geq 2$ \\
$(\tilde{\rightarrow} \mathrm{i}) \frac{\boldsymbol{q} Y}{\boldsymbol{q}(X \rightarrow Y)}$ & $(\tilde{\vee} \mathrm{i}) \frac{\boldsymbol{q} X}{\boldsymbol{q}(X \vee Y)} \quad \frac{\boldsymbol{q} Y}{\boldsymbol{q}(X \vee Y)}$ \\
& $(\tilde{\rightarrow} \mathrm{e}) \frac{\boldsymbol{q} X \quad \boldsymbol{q}(X \rightarrow Y)}{\boldsymbol{q} Y}$
\end{tabular}

Fig. 2. Calculus for SPIL. $\boldsymbol{q}$ ranges over quotation prefixes, $X, Y$ over formulas, and $S$ over conjunction sets.

SPIL is defined via the Hilbertian calculus given in Figure 2. The definitions of a derivation and its size are naturally adopted to this Hilbertian calculus. Note that derivations now consist of abstract formulas. We write $\Omega \vdash X$ to denote that the abstract formula $X$ has a derivation from the set $\Omega$ of abstract formulas in SPIL.

Definition 5. The consequence relation $\vdash$ between concrete formulas in SPIL is given by: $\Gamma \vdash x$ if $\{[y] \mid y \in \Gamma\} \vdash[x]$.

Note that the language of the concrete formulas is that of PIL. Abstract formulas are used only for defining this consequence relation.

Theorem 1. If $\Gamma$ entails $x$ in $\mathbf{P I L}$, then it does so in SPIL as well.

Next, we show that SPIL enjoys a locality property similar to that of PIL, which allows one to confine derivations of $X$ from $\Omega$ to those built from a certain small set of formulas computable from $X$ and $\Omega$. This property is essential for the correctness of the decision algorithm for SPIL.

Definition 6. The set of formulas that are local to a formula $X$ is inductively defined by: $(a) X$ is local to $X$; (b) If $\boldsymbol{q}(Y * Z)$ is local to $X$ (for $* \in\{\rightarrow, \vee\}$ and quotation prefix $\boldsymbol{q}$ ) then so are $\boldsymbol{q} Y$ and $\boldsymbol{q} Z$; and $(c$ ) If $\bigwedge S$ is local to $X$ (for conjunction set $S$ ) then so is every $Y \in S$. A formula is local to a set $\Omega$ of formulas if it is local to some $X \in \Omega$.

Definition 7. A derivation of a formula $X$ from a set $\Omega$ of formulas is called local if all node formulas of the derivation are local to $\Omega \cup\{X\}$.

Theorem 2. Any shortest derivation of $X$ from $\Omega$ in SPIL is local.

The following definition will be useful in the sequel. 
Definition 8. A quotation prefix $\boldsymbol{q}$ is local to a formula $X$ if some formula of the form $\boldsymbol{q} Y$ is local to $X$. A quotation prefix is local to a set $\Omega$ of formulas if it is local to some $X \in \Omega$.

\subsection{Semantics}

We adapt the semantics for PIL presented in [4] to SPIL.

Definition 9. A Kripke model is any structure $M$ whose vocabulary comprises of (i) binary relations $S_{q}$ where $q$ ranges over the principal constants and (ii) unary relations $V_{X}$ where $X$ ranges over non-conjunctive formulas. The elements of (the universe of) $M$ are called worlds.

Definition 10. Given a Kripke model $M$, we define when a world $w$ satisfies a formula $X$, symbolically $w \vDash X$, by induction on $s z(X)$, distinguishing the cases according to Proposition 2 ;

1. $X=[\top]: w \vDash X$ for every $w$.

2. $X=[v]$ (where $v$ is a propositional variable): $w \vDash X$ if $w \in V_{[v]}$.

3. $X=Y \rightarrow Z: w \vDash X$ if $w \vDash Z$ or $\left(w \not \models Y\right.$ and $\left.w \in V_{X}\right)$.

4. $X=Y \vee Z: w \vDash X$ if $w \vDash Y$ or $w \vDash Z$ or $w \in V_{X}$.

5. $X=q: Y$ (for non-conjunctive formula $Y$ ): $w \vDash X$ if $w^{\prime} \vDash Y$ for all $w^{\prime}$ with $w S_{q} w^{\prime}$.

6. $X=\bigwedge S: w \vDash X$ if $w \vDash Y$ for all $Y \in S$.

A world $w$ satisfies a set $\Omega$ of formulas if it satisfies every $X \in \Omega$.

Theorem 3 (Soundness and Completeness). Let $\Gamma$ be a set of concrete formulas and $x$ a concrete formula. $\Gamma \vdash x$ if and only if, for every Kripke model and world $w, w \vDash[x]$ whenever $w$ satisfies $\{[y] \mid y \in \Gamma\}$.

Remark 1. One of our referees wondered whether the full principle of replacement of equivalents holds in SPIL. It does not. Intuitively the reason is that, while SPIL generously enriches the algebra of conjunction, it imposes only mild restrictions on implication. Here is a example showing that the full principle of replacement of equivalents fails: $(x \wedge y) \rightarrow z \nvdash$ $(x \wedge(x \rightarrow y)) \rightarrow z$ while $x \wedge y$ and $x \wedge(x \rightarrow y)$ are interderivable. This can be easily verified using our Kripke semantics.

\section{A Decision Algorithm}

In this section we present an efficient decision algorithm for the the multiderivability problem for SPIL. 
Definition 11. The multi-derivability problem for SPIL is defined as follows. Given two sequences of concrete formulas, called concrete hypotheses and concrete queries respectively, decide which concrete queries are derivable from the concrete hypotheses in SPIL, and print them.

Theorem 4. There is a randomized algorithm that solves the multiderivability problem for SPIL in expected linear time and worst-case quadratic time.

Note that in "expected linear time" the average is taken for internal random choices during the execution, while assuming any input. We employ the same standard computation model of analysis of algorithms used in [4, according to which the registers are of size $O(\log n)$ where $n$ is the size of the input, and the basic register operations are constant time. We also presume a function Random that generates $\lceil\log (n)\rceil$ random bits in constant time.

The rest of this paper is devoted to prove Theorem 4 . The algorithm has two main stages. First, we construct a data structure that succinctly represents the input (Sections 4.1 and 4.2). Then, we use this data structure to compute the derivable concrete queries (Section 4.3).

\subsection{Input Parse Dag and Local Prefixes Dictionary}

We refer to the abstract formulas that correspond to the concrete hypotheses simply as hypotheses, and similarly to these of the concrete queries as queries. A formula (quotation prefix) is called a local input formula (local prefix) if it is local to the set of hypotheses or the set of queries (see Definitions 6 and 8). The input is represented in a directed acyclic graph (dag, for short) data structure ${ }^{6}$ We assume that each node $u$ is uniquely identified by a constant-size key, denoted by $\operatorname{Key}(u)$ (e.g., its memory address), stores the keys of its children in a list $\mathrm{Ch}(u)$, and of its parents in a corresponding list $\mathrm{Pa}(u)$. To handle quotation prefixes, we will use of the following auxiliary data structure:

Definition 12. A local prefixes dictionary for a given input is a data structure that assigns a unique constant-size $\operatorname{key} \operatorname{Key}(\boldsymbol{q})$ to every local input quotation prefix $\boldsymbol{q}$. Given such a key $k$, we will denote by $\operatorname{Prf}(k)$ the quotation prefix $\boldsymbol{q}$ such that $\operatorname{Key}(\boldsymbol{q})=k$.

Note that the trie of local prefixes as defined in [4] is a particular implementation of a local prefixes dictionary, where $\operatorname{Key}(\boldsymbol{q})$ is taken to

\footnotetext{
${ }^{6}$ By graph we actually mean multigraph, where parallel edges are allowed.
} 
be the memory address of the trie node that corresponds to $\boldsymbol{q}$. Given a local prefixes dictionary, our dag data structures are defined as follows.

Definition 13. A parse $d a g$ is a rooted dag in which every node $u$ is decorated with two additional (constant-size) fields: Label $(u)$ and $\operatorname{PrfKey}(u)$. Its root $r$ has two children denoted by $r_{h}$ and $r_{q}$, where $\operatorname{Label}(r)=\operatorname{Label}\left(r_{h}\right)=\operatorname{Label}\left(r_{q}\right)=\operatorname{nil}$ and $\operatorname{PrfKey}(r)=\operatorname{PrfKey}\left(r_{h}\right)=$ $\operatorname{PrfKey}\left(r_{q}\right)=\operatorname{Key}(\epsilon)$. All other nodes are called regular nodes. For each regular node $u, \operatorname{Label}(u)$ is $T, \rightarrow, \vee, \wedge$ or a propositional variable, and $\operatorname{PrfKey}(u)$ holds a key of some local input quotation prefix, such that:

1. If $\operatorname{Label}(u)$ is $T$ or a propositional variable, then $\mathrm{Ch}(u)$ is empty.

2. If Label $(u)$ is $\rightarrow$ or $\vee$, then $\mathrm{Ch}(u)$ contains exactly two keys.

3. If Label $(u)$ is $\wedge$, then $\mathrm{Ch}(u)$ contains at least one key.

4. If $u$ is a child of $v$, then $\operatorname{Prf}(\operatorname{PrfKey}(v))$ is a prefix of $\operatorname{Prf}(\operatorname{PrfKey}(u))$.

Each node in a parse dag naturally represents a (concrete and abstract) formula. Formally, this relation is defined as follows.

Notation 5 For a regular node $u$, we denote $\operatorname{Prf}(\operatorname{PrfKey}(u))$ by $\operatorname{Prf}(u)$.

Notation 6 Given two quotation prefixes $\boldsymbol{q}$ and $\boldsymbol{p}$, we denote by $\boldsymbol{p} \backslash \boldsymbol{q}$ the quotation prefix $\boldsymbol{r}$, such that $\boldsymbol{p r}=\boldsymbol{q}$, or $\epsilon$ if such $\boldsymbol{r}$ does not exist.

Definition 14. The complete concrete formula of a regular node $u$ with respect to a quotation prefix $\boldsymbol{q}$ is denoted by $\mathrm{F}(u, \boldsymbol{q})$, and defined by:

1. If $u$ has no children, then $\mathrm{F}(u, \boldsymbol{q})=(\boldsymbol{q} \backslash \operatorname{Prf}(u)) \operatorname{Label}(u)$.

2. If $\operatorname{Label}(u)=*$ for $* \in\{\rightarrow, \vee\}$, then $\mathrm{F}(u, \boldsymbol{q})$ is $(\boldsymbol{q} \backslash \operatorname{Prf}(u))\left(\mathrm{F}\left(u_{1}, \operatorname{Prf}(u)\right) * \mathrm{~F}\left(u_{2}, \operatorname{Prf}(u)\right)\right) \quad$ where $\quad u_{1} \quad$ and $\quad u_{2} \quad$ are the first and second children of $u$ (respectively).

3. If $\operatorname{Label}(u) \quad=\wedge$, then $\mathrm{F}(u, \boldsymbol{q})$ is $(\boldsymbol{q} \backslash \operatorname{Prf}(u))\left(\left(\cdots\left(\mathrm{F}\left(u_{1}, \operatorname{Prf}(u)\right) \wedge \mathrm{F}\left(u_{2}, \operatorname{Prf}(u)\right)\right) \ldots\right) \wedge \mathrm{F}\left(u_{k}, \operatorname{Prf}(u)\right)\right)$ where $u_{l}, \ldots, u_{k}$ are $u$ 's children in the order they occur in $\mathrm{Ch}(u)$.

The complete concrete formula of a regular node $u$ is denoted by $\mathrm{F}(u)$ and defined to be $\mathrm{F}(u, \epsilon)$. The complete (abstract) formula of a regular node $u$ is denoted by $\tilde{\mathrm{F}}(u)$ and defined to be $[\mathrm{F}(u)]$.

Definition 15. A parse dag for input $x_{1}, \ldots, x_{k} \vdash y_{1}, \ldots, y_{m}$ is any parse dag that satisfies the following conditions: $\quad(1) \quad\left\{\tilde{\mathrm{F}}(u) \mid \operatorname{Key}(u) \in \operatorname{Ch}\left(r_{h}\right)\right\}=\left\{\left[x_{1}\right], \ldots,\left[x_{k}\right]\right\}$; $\left\{\tilde{\mathrm{F}}(u) \mid \operatorname{Key}(u) \in \operatorname{Ch}\left(r_{q}\right)\right\}=\left\{\left[y_{1}\right], \ldots,\left[y_{m}\right]\right\} ;$ and (3) Every child $u$ of $r_{q}$ is decorated with a list $\operatorname{Inputs}(u)$ of all $y_{i}$ 's that satisfy $y_{i} \in \tilde{\mathrm{F}}(u)$. 
Note that the input parse tree as defined in [4] (ignoring the edge labels) is also an input parse dag. For the next stage, we should ensure that there are no two different nodes that represent the same formula. Thus we are interested in a compressed input parse dag, as defined next.

Definition 16. A node $u$ in a parse dag $D$ is unique if $\tilde{\mathrm{F}}\left(u^{\prime}\right) \neq \tilde{\mathrm{F}}(u)$ for any $u^{\prime} \neq u$. D is called compressed if its nodes are all unique, and $\operatorname{Label}(u)$ is not $\wedge$ or $\top$ whenever $u$ is a child of a node labeled with $\wedge$.

Proposition 3. Consider a compressed input parse dag. For every local input formula $X$, there is exactly one regular node $u$ such that $\tilde{F}(u)=X$.

Theorem 7. There is a randomized algorithm with expected linear time and worst-case quadratic time complexities, that constructs a local prefixes dictionary and a compressed input parse dag for a given input.

\subsection{Construction of a Compressed Input Parse Dag}

This section is devoted to prove Theorem 7. To facilitate the exposition and the analysis, we will present this algorithm as a composition of sub-algorithms. Initially, we construct (in linear time) a local prefixes dictionary and an initial (uncompressed) input parse dag (in the form of a trie of local prefixes and an input parse tree) exactly as done in [4]. It remains to modify the parse tree into a compressed parse dag. First, we reformat the tree as detailed in Algorithm 4.1. Roughly speaking, this step accounts for the associativity of conjunction. Its time complexity is $O(N)$, where $N$ denotes the number of leaves in the initial parse tree.

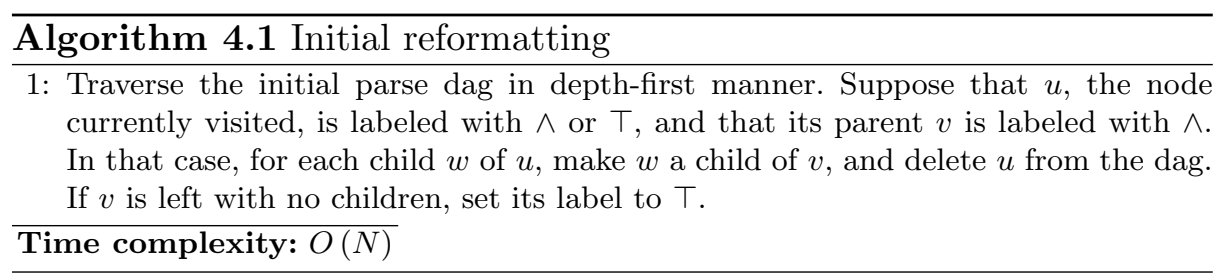

Next, we "compress" the resulting tree into a dag. This process requires several additional data structures and fields:

1. A work list $C$ of length $N$, initialized with (the keys of) all leaf nodes.

2. Two auxiliary arrays $A$ and $H T$ (Hash Table) of length $M=$ $2^{\left\lceil\log _{2}(N)\right\rceil}$. The entries of $A$ are node keys, while those of $H T$ are lists of keys. Initially $A[i]=-1$ and $H T[i]=\emptyset$ for all $i<M$. 
3. Numeric fields $\operatorname{Counter}(u)$ and $\operatorname{Hash}(u)$ for each node $u$, initialized with 0 and a random number $<M$ (respectively).

The compression works iteratively using the work list $C$. In each iteration, the nodes in $C$ are made unique. Initially, $C$ includes all leaf nodes. When a node $u$ is removed from $C$, it increments the counter in its parent $v$. If Counter $(v)$ reaches $|\mathrm{Ch}(v)|$ (the length of the list $\mathrm{Ch}(v)$ ), $v$ is added to $C$ for the next iteration. Thus, the following invariant is preserved:

Invariant 8 Children of nodes in $C$ are all unique, and each node in $C$ or ancestor of a node in $C$ has a unique parent node.

Compression of the leaves. Before the first iteration, the work list $C$ includes all leaf nodes. They are compressed using a plagiarism checker:

Definition 17. An element $a_{j}$ in an array $L=\left(a_{0}, \ldots, a_{k-1}\right)$ is original if $a_{i} \neq a_{j}$ for any $i<j$. If $a_{i}$ is original, $i \leq j$ and $a_{i}=a_{j}$ then $a_{i}$ is the original of $a_{j}$. A plagiarism checker for the array $L$ is an array $B$ of length $k$ such that every $a_{B[j]}$ is the original of $a_{j}$.

Theorem 9. There is an algorithm that, given an array $L$ of d-tuples of natural numbers $<M$ and an array $A$ of length $M$ initialized with (-1)'s, computes the plagiarism checker $B$ for $L$ and re-initializes the array $A$ with (-1)'s (so it can be reused to compute future plagiarism checkers). This algorithm takes $O(|L| d)$ time.

The plagiarism checker is computed on an array $L$ that includes the extended labels of the leaf nodes. For each node $u$ in $C$, the extended label of $u$, denoted by $\operatorname{EL}(u)$, is a constant-size tuple that satisfies the following property: for every two nodes $u_{1}$ and $u_{2}$ in $C, \operatorname{EL}\left(u_{1}\right)=\operatorname{EL}\left(u_{2}\right)$ iff $\tilde{\mathrm{F}}\left(u_{1}\right)=\tilde{\mathrm{F}}\left(u_{2}\right)$. For a leaf node $u$, the extended label $\mathrm{EL}(u)$ is taken to be the ordered pair $(\operatorname{PrfKey}(u), \operatorname{Label}(u))$ if $\operatorname{Label}(u)$ is a propositional variable, or just $T$ if $\operatorname{Label}(u)=T$. It is easy to see that this definition of $\mathrm{EL}(u)$ guarantees the required property for the (leaf) nodes in $C$. From this observation, Algorithm 4.2 for compression of the leaves follows. Note that computing the extended label of a leaf takes constant time. Thus computing $L$ and $U$ takes $O(N)$ time. Since extended labels have constant length, the plagiarism checker can be computed in $O(N)$ time as well.

Compression of internal nodes. After applying Algorithm 4.2 in the first iteration, all leaf nodes are unique. In addition, Algorithm 4.2 prepares it for the next iteration, so it includes all nodes whose children are 


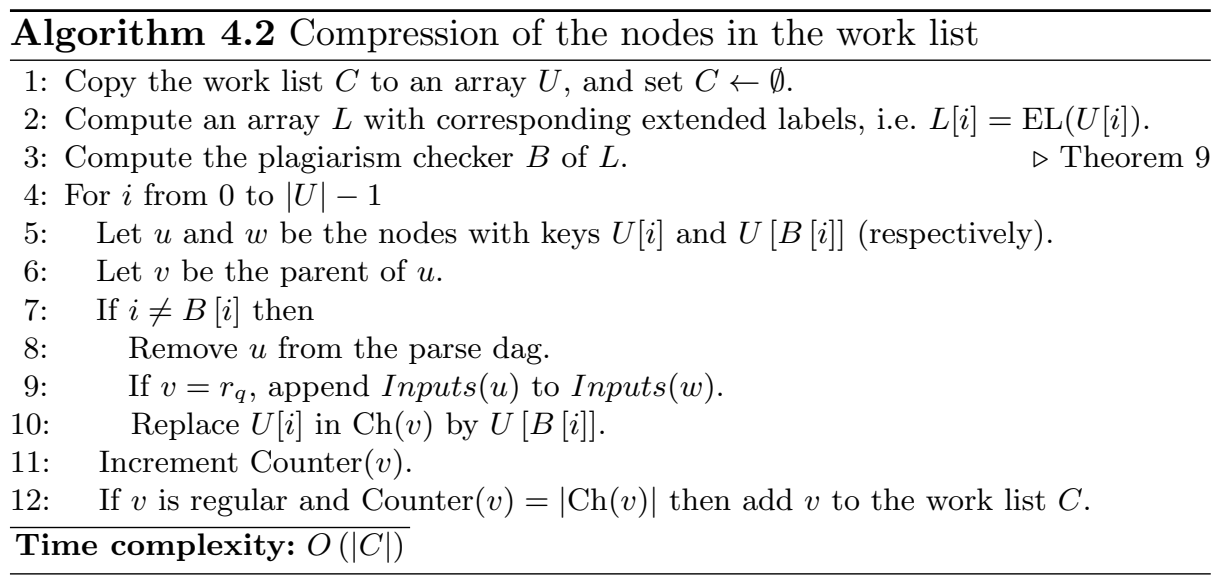

all unique. In fact, the next iteration also applies Algorithm 4.2, with a different definition of the extended labels. We refer to the nodes of $C$ whose label is $\rightarrow$ or $\vee$ as binary nodes, and to these whose label is $\wedge$ as set nodes. The extended label for the binary nodes is simple, as we can take $\operatorname{EL}(u)$ of a binary node $u$ in $C$ with first child $v$ and second child $w$ to be the ordered tuple $(\operatorname{PrfKey}(u), \operatorname{Label}(u), \operatorname{Key}(v), \operatorname{Key}(w))$.

Proposition 4. For two binary nodes $u_{1}$ and $u_{2}$ in $C, \operatorname{EL}\left(u_{1}\right)=\operatorname{EL}\left(u_{2}\right)$ iff $\tilde{F}\left(u_{1}\right)=\tilde{F}\left(u_{2}\right)$.

The compression of the set nodes, however, is more involved, and requires some preprocessing to account for the idempotence of $\wedge$, and to compute the extended labels of the set nodes. Several additional notations are used in this preprocessing stage:

- $N_{C}$ denotes the sum $\sum|\mathrm{Ch}(u)|$ for all set nodes in $C$.

- For each set node $u$ in $C, C H(u)=\{w \mid w$ is a child of $u\}$.

The preprocessing for the set nodes consists of two steps. First, we reformat the parse dag, by removing duplicate children of set nodes, as well as contracting set nodes that are left with only one child (this may add new binary nodes to $C$ ). Algorithm 4.3 provides the technical details. Intuitively, this step accounts for the idempotence of $\wedge$.

Next, we compute the extended labels for the set nodes. This step involves a hash table, where the hash function assigns to each node $u$ the initially chosen random number $\operatorname{Hash}(u)$. Note that $(\wedge, \mathrm{Ch}(u))$ cannot serve as an extended label (since two set nodes with different permutations of the same list of children would have diffrent extended labels). 


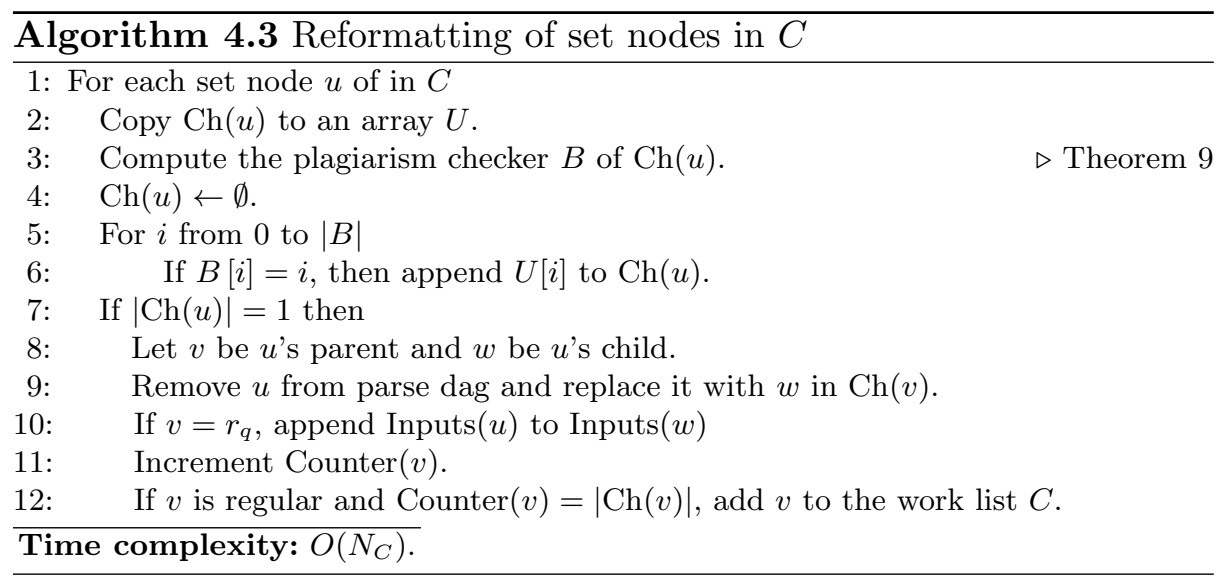

Compute extended labels. We assume that each set node $u$ is decorated with an additional field called set label and denoted by SL $(u)$. For each set node $u$ in $C, \operatorname{SL}(u)$ is initialized with $\operatorname{Hash}\left(u_{1}\right) \oplus \cdots \oplus \operatorname{Hash}\left(u_{k}\right)$, where $u_{1}, \ldots, u_{k}$ are the children of $u$ whose keys are listed in $\mathrm{Ch}(u)$, and $\oplus$ is the bitwise XOR operation (between binary representations of numbers). The computation of $\mathrm{SL}(u)$ takes $O(|\mathrm{Ch}(u)|)$ time for each node $u$. Hence the computation for all set nodes in $C$ takes $O\left(N_{C}\right)$ time. Note that for two set nodes $u$ and $v$ with $C H(u)=C H(v)$, we have $\mathrm{SL}(u)=\mathrm{SL}(v)$; the converse, however, is "almost always" true as the following lemma shows.

Lemma 1. For every two set nodes, $P(\mathrm{SL}(u)=\mathrm{SL}(v))$ is 1 if $C H(u)=$ $C H(v)$, and $1 / M$ otherwise.

It follows that SL cannot serve as an extended label for the set nodes. To generate the extended labels EL, we use a hash table for detecting and fixing collisions in SL. This is described in Algorithm 4.4. We explain the time complexity for this computation. Let $u_{1}, u_{2}, \ldots, u_{|C|}$ be the set nodes in $C$ and suppose that the loop in line 1 process them in that order. For $i<j \leq|C|$, let $X_{i j}$ be a random variable which takes value 1 , if EL $\left(u_{i}\right)=$ $\left(\wedge, \operatorname{Key}\left(u_{i}\right)\right)$ and $\operatorname{SL}\left(u_{i}\right)=\operatorname{SL}\left(u_{j}\right)$; and 0 , otherwise. Let $T$ be the random variable that gives the time complexity for this computation. $T$ is the sum over $j$ of the time needed to compute $\operatorname{EL}\left(u_{j}\right)$. To compute $\operatorname{EL}\left(u_{j}\right)$ we check the nodes in the entry $H T$ [SL $\left.\left(u_{j}\right)\right]$. The length of $H T$ [SL $\left.\left(u_{j}\right)\right]$ is $\sum_{i=1}^{j-1} X_{i j}$ and each comparison (the check if $C H(u)=C H(v)$ ) takes $O\left(\left|\mathrm{Ch}\left(u_{j}\right)\right|\right)$ time. Therefore, $T=\sum_{j=1}^{|C|}\left(O\left(\left|\mathrm{Ch}\left(u_{j}\right)\right|\right) \cdot \sum_{i=1}^{j-1} X_{i j}\right)$. In the worst-case, for any two set nodes $u$ and $v$ we have $\mathrm{SL}(u)=\mathrm{SL}(v)$ but $C H(u) \neq C H(v)$. This yields an execution time of $O\left(|C| \cdot N_{C}\right)$. To see 
that $E(T)=O\left(N_{C}\right)$, it suffices to show that $\sum_{i=1}^{j-1} E\left(X_{i j}\right)$ is constant. Now, $E\left(X_{i j}\right)=P\left(X_{i j}=1\right) \leq P\left(\mathrm{SL}\left(u_{i}\right)=\mathrm{SL}\left(u_{j}\right)\right)$, and by Lemma 1 we obtain that $E\left(X_{i j}\right) \leq 1$ if $C H\left(u_{i}\right)=C H\left(u_{j}\right)$, and $E\left(X_{i j}\right) \leq 1 / M$ otherwise. Algorithm 4.4 stores at most one set node $u_{i}$ with $C H\left(u_{i}\right)=C H\left(u_{j}\right)$ in $H T\left[\operatorname{SL}\left(u_{j}\right)\right]$. Hence, $\sum_{i=1}^{j-1} E\left(X_{i j}\right) \leq 1+\frac{j-2}{M} \leq 1+\frac{N}{M} \leq 2$.

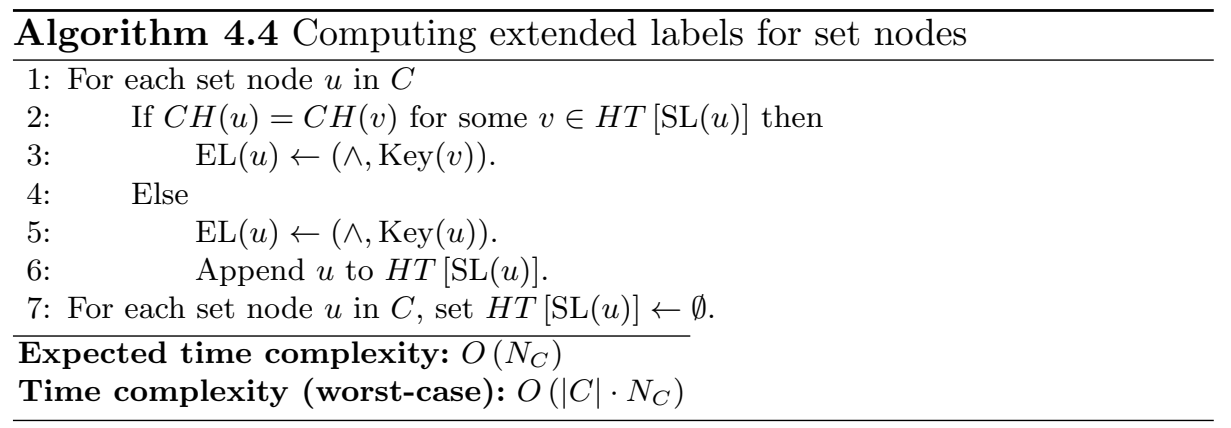

Compression of internal nodes. Equipped with extended labels for all nodes in the work list $C$, we apply the compression for these nodes. Since each two nodes $u_{1}$ and $u_{2}$ in $C$ have $\tilde{\mathrm{F}}\left(u_{1}\right)=\tilde{\mathrm{F}}\left(u_{2}\right)$ iff $\operatorname{EL}\left(u_{1}\right)=\operatorname{EL}\left(u_{2}\right)$, we can compress the nodes in $C$ exactly as we did for the leaves using Algorithm 4.2. This algorithm also prepares $C$ for the next iteration.

This concludes the computation of a compressed parse dag from the parse tree. Algorithm 4.5 gives a summary of this construction. To see the time complexity of Algorithm 4.5, note that the inner step of the loop takes expected time proportional to the number of nodes in $C$ plus the number of their children. Since every node is added to $C$ exactly one time, summing this over all iterations we get expected time proportional to the number of nodes. In a similar way, we get $O\left(N^{2}\right)$ time in the worstcase for the inner loop. The complexities of all other steps were explained above. Finally, note that $N$ (the number of leaves in the initial input tree) is clearly less than the length of the input.

\subsection{Deriving Local Formulas}

The second stage of algorithm computes all derivable queries. This is done similarly to the corresponding stage for PIL [4]. First, we traverse the parse dag and decorate each regular node $u$ with a boolean flag $\operatorname{Der}(u)$. It is initialized to 0 , unless $\operatorname{Label}(u)=\top$ or $u$ represents a hypothesis ( $u$ is a child of $r_{h}$ ) in which case $\operatorname{Der}(u)$ is initialized to 1 . $\operatorname{Der}(u)=0$ 


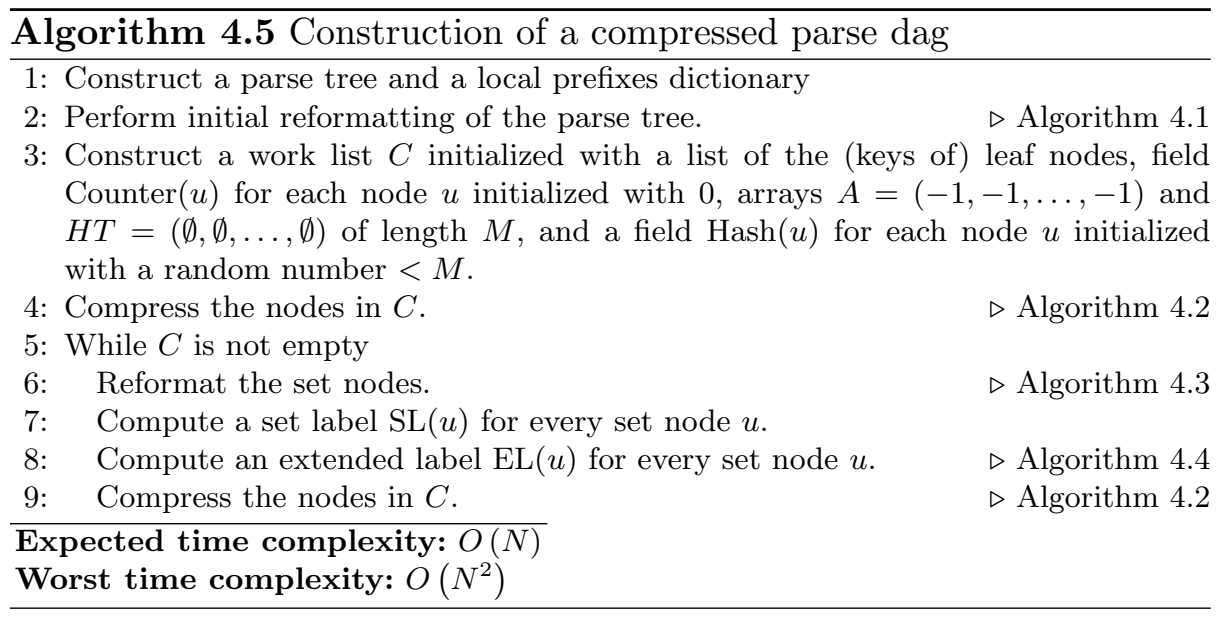

indicates that $\tilde{\mathrm{F}}(u)$ has not been derived from the hypotheses yet, and in this case we say that $u$ is $\operatorname{raw}$. $\operatorname{Der}(u)=1$ indicates that $\tilde{\mathrm{F}}(u)$ has been derived from the hypotheses, and $u$ is called pending. We also construct a pending queue, that contains all pending nodes. To make pending a node $u$ means to insert $u$ to the pending queue and set $\operatorname{Der}(u)=1$. The following invariant holds throughout the execution of the algorithm.

Invariant 10 Whenever a node $u$ becomes pending, the formula $\tilde{F}(u)$ is derivable from the hypotheses in SPIL.

To apply a rule $R$ to $u$ means to make pending every raw node $w$ for which there are pending nodes $v_{1}, v_{2}, \ldots, v_{k}$, such that $u \in\left\{v_{1}, v_{2}, \ldots, v_{k}\right\}$ and $\tilde{\mathrm{F}}\left(v_{1}\right), \ldots, \tilde{\mathrm{F}}\left(v_{k}\right) / \tilde{\mathrm{F}}(w)$ is an instance of the rule $R$. The algorithm repeatedly takes a node $u$ from the pending queue, applies as many rules to it as possible and then removes $u$ from the pending queue. The algorithm terminates when the pending queue is empty. We explain how to apply each rule $R$ to a node $u$, and show (for several cases) that these applications preserve Invariant 10. Note that an additional numeric field Counter $(u)$ (initialized to 0 ) is used for each node $u$ labeled with $\wedge$.

( $\tilde{\wedge} \mathbf{e})$ If Label $(u)=\wedge$, then make pending every raw child of $u$.

Justification: Let $u_{1}, \ldots, u_{k}$ be the children of $u$ in the order they appear in $\operatorname{Ch}(u)$. Then $\tilde{\mathrm{F}}(u)=\left[\operatorname{Prf}(u)\left(\left(\cdots\left(\mathrm{F}\left(u_{1}, \operatorname{Prf}(u)\right) \wedge\right.\right.\right.\right.$ $\left.\left.\left.\mathrm{F}\left(u_{2}, \operatorname{Prf}(u)\right) \ldots\right) \wedge \mathrm{F}\left(u_{k}, \operatorname{Prf}(u)\right)\right)\right]$. Since $u$ is pending, $\tilde{\mathrm{F}}(u)$ is derivable. This entails that $\tilde{\mathrm{F}}\left(u_{i}\right)=\left[\operatorname{Prf}(u)\left(\mathrm{F}\left(u_{i}, \operatorname{Prf}(u)\right)\right]\right.$ is derivable as well. To see this, note that $\boldsymbol{q} x_{i}$ is derivable from $\boldsymbol{q}\left(\left(\cdots\left(x_{1} \wedge x_{2}\right) \ldots\right) \wedge\right.$ 
$x_{k}$ ) in PIL for every concrete formulas $x_{1}, \ldots, x_{k}$, quotation prefix $\boldsymbol{q}$, and $1 \leq i \leq k$. By Theorem 1 , we have that $\tilde{\mathrm{F}}(u) \vdash \tilde{\mathrm{F}}\left(u_{i}\right)$.

$(\tilde{\wedge} \mathbf{i})$ For every raw parent $w$ of $u$ labeled with $\wedge$, increment Counter $(w)$ and make $w$ pending if it exceeds the number of children of $w$.

Justification: Let $u_{1}, \ldots, u_{k}$ be the children of $w$ in the order they appear in $\operatorname{Ch}(w)$. Then $\tilde{\mathrm{F}}(w)=\left[\operatorname{Prf}(w)\left((\cdots)\left(\mathrm{F}\left(u_{1}, \operatorname{Prf}(w)\right) \wedge\right.\right.\right.$ $\left.\left.\left.\mathrm{F}\left(u_{2}, \operatorname{Prf}(w)\right) \ldots\right) \wedge \mathrm{F}\left(u_{k}, \operatorname{Prf}(w)\right)\right)\right]$. If Counter $(w)$ was incremented $k$ times, then each $\tilde{\mathrm{F}}\left(u_{i}\right)=\left[\operatorname{Prf}(w)\left(\mathrm{F}\left(u_{i}, \operatorname{Prf}(w)\right)\right]\right.$ is derivable. This entails that $\tilde{\mathrm{F}}(w)$ is derivable as well (again using Theorem 1, since $\boldsymbol{q}\left(\left(\cdots\left(x_{1} \wedge x_{2}\right) \ldots\right) \wedge x_{k}\right)$ is derivable from $\boldsymbol{q} x_{1}, \ldots, \boldsymbol{q} x_{k}$ in PIL for every concrete formulas $x_{1}, \ldots, x_{k}$ and quotation prefix $\left.\boldsymbol{q}\right)$.

( $\tilde{\nabla} \mathbf{i})$ Make pending every raw parent $w$ of $u$ labeled with $\vee$.

$(\tilde{\rightarrow} \mathbf{i})$ For every raw parent $w$ of $u$ such that $\operatorname{Label}(w)$ is $\rightarrow$ and $u$ is the second child of $w$, make $w$ pending.

$(\tilde{\rightarrow} \mathbf{e}) \tilde{\mathrm{F}}(u)$ can be used as the left or the right premise of $(\tilde{\rightarrow} \mathrm{e})$. Accordingly, we have two substeps: (1) For every pending parent $w$ of $u$, such that $\operatorname{Label}(w)$ is $\rightarrow$ and $u$ is the first child of $w$, make the second child of $w$ pending if it is raw; (2) If $\operatorname{Label}(u)$ is $\rightarrow$ and the first child $u_{1}$ of $u$ is pending, then make pending the second child $u_{2}$ of $u$ if it is raw.

When the pending queue is empty, the algorithm prints a list of the derivable concrete queries. To do so, walk through the nodes $u_{1}, \ldots, u_{m}$ that represent queries (i.e. the children of the node $r_{q}$ ). If $\operatorname{Der}\left(u_{i}\right)=1$ then print the strings in Inputs $\left(u_{i}\right)$. Since separate concrete queries are separate segments of the input, the printing process takes linear time.

Theorem 11. The decision algorithm for SPIL is sound and complete, and it works in expected linear time and quadratic time in the worst-case.

\section{References}

1. Lev Beklemishev and Yuri Gurevich. Propositional primal logic with disjunction. Journal of Logic and Computation, 24(1):257-282, 2014.

2. Lev Beklemishev and Igor Prokhorov. On computationally efficient subsystems of propositional logic. To appear.

3. Andreas Blass, Guido De Caso, and Yuri Gurevich. An Introduction to DKAL. Microsoft Research technical report, MSR-TR-2012-108, 2012.

4. Carlos Cotrini and Yuri Gurevich. Basic primal infon logic. Journal of Logic and Computation, 2013.

5. Carlos Cotrini and Yuri Gurevich. Transitive primal infon logic. The Review of Symbolic Logic, 6:281-304, 2013.

6. Gilles Dowek, Thérèse Hardin, and Claude Kirchner. Theorem proving modulo. J. Autom. Reason., 31(1):33-72, 2003.

7. Yuri Gurevich and Itay Neeman. Logic of infons: The propositional case. $A C M$ Trans. Comput. Logic, 12(2):9:1-9:28, 2011. 